\title{
Formation Dynamics of Carbon Atomic Chain from Graphene by Electron Beam Irradiation
}

\author{
Hyo Ju Park, Zonghoon Lee*
}

School of Materials Science and Engineering, Ulsan National Institute of Science and Technology (UNIST), UIsan 44919, Korea

*Correspondence to:

Lee $\mathrm{Z}$,

(iD) http://orcid.org/0000-0003-3246-4072

Tel: $+82-52-217-2327$

Fax: +82-52-217-2309

E-mail: zhlee@unist.ac.kr

Received December 20, 2018

Revised December 21, 2018

Accepted December 21, 2018
Carbon has numerous allotropes and various crystalline forms with full dimensionalities such as diamond, graphite, fullerenes, and carbon nanotubes leading a wide range of applications. Since the emerge of graphene consisting of a single atomic layer of carbon atoms, a fabrication of all-carbon-based device with combination of one-, two-, and three-dimensional carbons has become a hot issue. Here, we introduce an ultimate onedimensional carbon atomic chain. Carbon atomic chains were experimentally created by removing atoms from monolayer graphene sheet under electron beam inside transmission electron microscope (TEM). A series of TEM images demonstrate the dynamics of carbon atomic chains over time from the formation, transformation, and then breakage.

Key Words: Aberration-corrected transmission electron microscope, Carbon atomic chain, Graphene, Defect structure, Electron beam irradiation
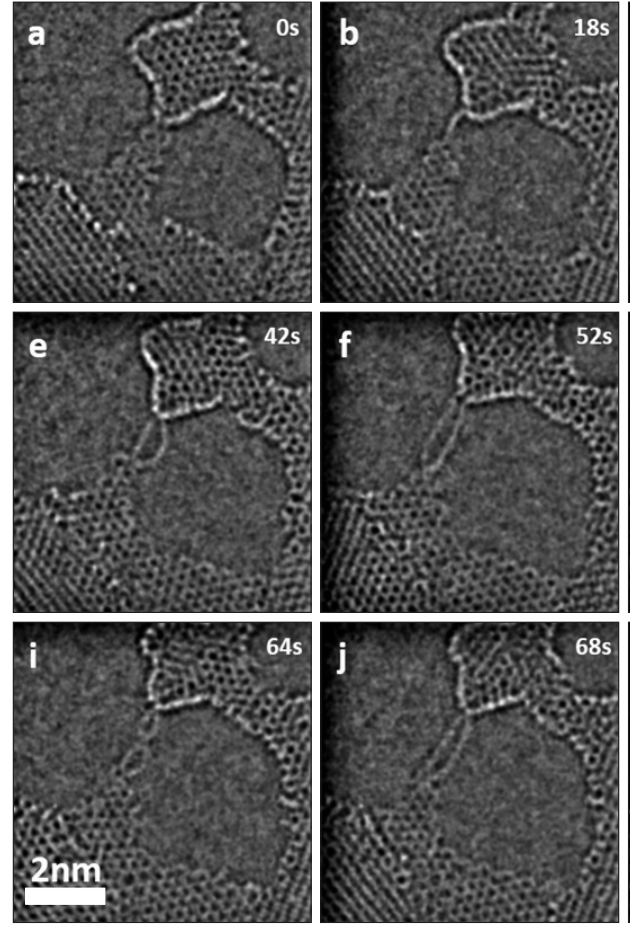
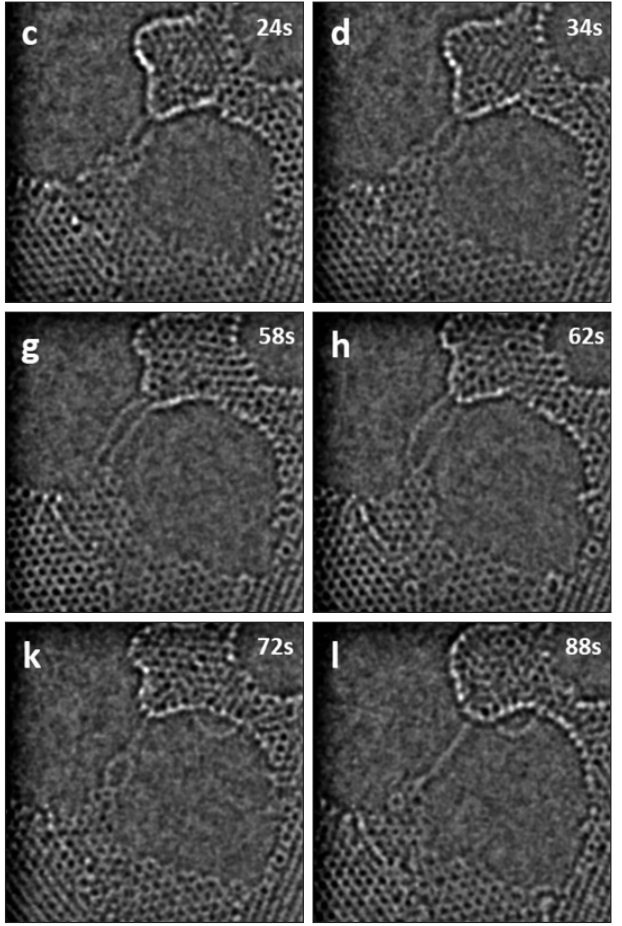

(a) This is an open-access article distributed under the terms of the Creative Commons Attribution Non-Commercial License (http://creativecommons.org/licenses/by-nc/4.0) which permits unrestricted noncommercial use, distribution, and reproduction in any medium, provided the original work is properly cited.

Copyrights (c) 2018 by Korean Society of Microscopy 
Formation and dynamics of carbon atomic chains were investigated using an aberration-corrected transmission electron microscope (FEI Titan ${ }^{3}$ G2 60-300) at $80 \mathrm{kV}$. Imaging condition was settled as $-21 \pm 0.5 \mu \mathrm{m}$ of spherical aberration $\left(\mathrm{C}_{\mathrm{s}}\right.$ ), around $5 \times 10^{5}$ $\mathrm{e}^{-} \mathrm{nm}^{-2}$ of electron beam density. Time-lapse images were acquired over 88 seconds using Gatan DigitalMicrograph script and the atomic structures were analyzed frame-by-frame. Each of the images was acquired with an exposure time of $0.3 \mathrm{~s}$, and the interval time between frames were $1.7 \mathrm{~s}$.

The figure shows the formation, transformation and breakage of carbon atomic chain derived from monolayer graphene by prolonged electron beam irradiation. Firstly, carbon atoms were knocked off from a monolayer graphene sheet and graphene nanoribbon was formed (a). Additional ejection of atoms results in double-strand of carbon atomic chains $(\mathrm{c} \sim \mathrm{h})$. Temporal twist of double-strand was also observed (i). Finally, the end of one chain was detached from the graphene sheet and bound to the other chain $(\mathrm{k})$ and then broken, leaving single carbon atomic chain (l). Although both carbon atomic chains were eventually broken by additional electron beam irradiation, they endured the intense electron beam over tens of seconds. This figure shows the carbon atomic chains are quite durable and flexible under electron beam (Banhart, 2015; Chuvilin et al., 2009; Jin et al., 2009).

\section{CONFLICT OF INTEREST}

No potential conflict of interest relevant to this article was reported.

\section{ACKNOWLEDGMENTS}

The authors acknowledge the funding support from National Research Foundation of Korea (NRF) grant funded by the Korea government (MSIT; no. 2018R1A2A2A05019598).

\section{REFERENCES}

Banhart F (2015) Chains of carbon atoms: A vision or a new nanomaterial? Beilstein Journal of Nanotechnology 6, 559-569.

Chuvilin A, Meyer J C, Algara-Siller G, and Kaiser U (2009) From graphene constrictions to single carbon chains. New Journal of Physics 11,
083019.

Jin C H, Lan H P, Peng L M, Suenaga K, and lijima S (2009) Deriving Carbon Atomic Chains from Graphene. Physical Review Letters 102, 205501. 